\title{
Universiteit
}

Leiden

The Netherlands

\section{Independent tuning of quantum dots in a photonic crystal cavity}

Kim, H.; Thon, S.M.; Petroff, P.M.; Bouwmeester, D.

\section{Citation}

Kim, H., Thon, S. M., Petroff, P. M., \& Bouwmeester, D. (2009). Independent tuning of quantum dots in a photonic crystal cavity. Applied Physics Letters, 95, 243107. doi:10.1063/1.3275002

Version: $\quad$ Not Applicable (or Unknown)

License: $\quad$ Leiden University Non-exclusive license

Downloaded from: https://hdl.handle.net/1887/65883

Note: To cite this publication please use the final published version (if applicable). 


\section{Independent tuning of quantum dots in a photonic crystal cavity}

Hyochul Kim, Susanna M. Thon, Pierre M. Petroff, and Dirk Bouwmeester

Citation: Appl. Phys. Lett. 95, 243107 (2009); doi: 10.1063/1.3275002

View online: https://doi.org/10.1063/1.3275002

View Table of Contents: http://aip.scitation.org/toc/apl/95/24

Published by the American Institute of Physics

\section{Articles you may be interested in}

Strong coupling through optical positioning of a quantum dot in a photonic crystal cavity Applied Physics Letters 94, 111115 (2009); 10.1063/1.3103885

Independent electrical tuning of separated quantum dots in coupled photonic crystal cavities Applied Physics Letters 99, 161102 (2011); 10.1063/1.3651491

Permanent tuning of quantum dot transitions to degenerate microcavity resonances Applied Physics Letters 98, 121111 (2011); 10.1063/1.3569587

Deterministic nanoassembly of a coupled quantum emitter-photonic crystal cavity system Applied Physics Letters 98, 193103 (2011); 10.1063/1.3571437

Tuning micropillar cavity birefringence by laser induced surface defects Applied Physics Letters 95, 251104 (2009); 10.1063/1.3276550

Fiber-connectorized micropillar cavities Applied Physics Letters 97, 131113 (2010); 10.1063/1.3493187

\section{Conference Proceedings}

Get $30 \%$ off all print proceedings!
Enter Promotion Code PDF-30 at checkout

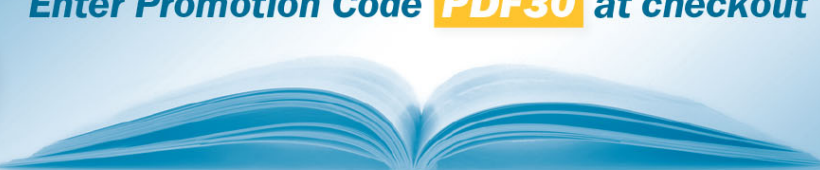




\title{
Independent tuning of quantum dots in a photonic crystal cavity
}

\author{
Hyochul Kim, ${ }^{1, a)}$ Susanna M. Thon, ${ }^{1}$ Pierre M. Petroff, ${ }^{2,3}$ and Dirk Bouwmeester ${ }^{1,4}$ \\ ${ }^{1}$ Department of Physics, University of California-Santa Barbara, Santa Barbara, California 93106, USA \\ ${ }^{2}$ Department of Materials, University of California-Santa Barbara, Santa Barbara, California 93106, USA \\ ${ }^{3}$ Department of Electrical and Computer Engineering, University of California-Santa Barbara, \\ Santa Barbara, California 93106, USA \\ ${ }^{4}$ Huygens Laboratory, Leiden University, P.O. Box 9504, 2300 RA Leiden, The Netherlands
}

(Received 27 September 2009; accepted 22 November 2009; published online 15 December 2009)

\begin{abstract}
One of the main obstacles to coupling two quantum dots (QDs) to a single nanocavity mode in a cavity quantum electrodynamics system is the ability to independently tune the QD frequencies. We demonstrate that in a GaAs photonic crystal membrane structure with two embedded QD layers, the QD emission frequencies of one QD layer can be tuned independently of the other by applying a voltage across only one of the QD layers. (c) 2009 American Institute of Physics.
\end{abstract}

[doi:10.1063/1.3275002]

Self assembled quantum dots (QDs) embedded in optical nanocavities are an attractive system for solid state cavity quantum electrodynamics experiments and offer the potential for the implementation of quantum information (QI) processing schemes. Strong coupling between a single QD and a nanocavity has been recently demonstrated. ${ }^{1-5}$ A crucial next step toward making these systems useful for QI purposes is to induce coherent interaction among individual QD-cavity systems. For example, two QDs can be strongly coupled to a single cavity mode, resulting in the generation of tripartite quantum states, ${ }^{6}$ or two individual QD-cavity systems could be entangled through interactions via a single optical channel. $^{7-9}$

The main challenges in achieving control of multiple QD systems are the random spatial nucleation and large variation in emission frequencies of the self-assembled QDs. ${ }^{10}$ There have been several solutions to the QD-cavity mode spatial matching problem, which include using QD stacks as indicators of seed QD positions, ${ }^{11}$ measuring the positions of QDs using the surface morphology of GaAs due to the strain induced by the QDs ${ }^{4}$ and using an optical method to premeasure QD positions. ${ }^{12,13}$ Several methods have been used to spectrally tune the QD emission into resonance with a cavity mode, such as temperature tuning ${ }^{2,14}$ and Stark shift tuning. ${ }^{15}$ In addition, depositing gas monolayers ${ }^{16,17}$ or irreversibly etching photonic crystal membranes ${ }^{18}$ can be used to tune the cavity mode frequency.

Due to the small linewidths of the QDs and cavity modes and the frequency spread of the QD ensemble, the tuning of individual QD frequencies is necessary to achieve coupling between two closely spaced QDs and a photonic crystal cavity mode. In this letter, we report on the independent tuning of QDs in separate layers in a photonic crystal cavity. Two layers of QDs are grown in a GaAs membrane suitable for photonic crystal fabrication, and a direct current $(\mathrm{dc})$ electric field is introduced across only one of the QD layers. The emission of this layer of QDs is tuned using the Stark effect, while the second closely spaced layer of QDs is unaffected.

The sample consists of a $0.92 \mu \mathrm{m} \mathrm{Al}_{0.7} \mathrm{Ga}_{0.3}$ As sacrificial layer grown on a semi-insulating GaAs substrate followed by a $190 \mathrm{~nm}$ GaAs membrane structure. In the GaAs

${ }^{a)}$ Electronic mail: hckim@physics.ucsb.edu. membrane, two layers of QDs and two dopant layers are introduced. The structure of the membrane and the doping concentration are schematically described in Fig. 1(a). The two QD layers are located $40 \mathrm{~nm}$ above and $50 \mathrm{~nm}$ below the membrane center. Two layers of $\mathrm{In}_{0.4} \mathrm{Ga}_{0.6} \mathrm{As}$ QDs are grown by depositing ten periods of $0.55 \AA$ of InAs and $1.2 \AA$ of $\mathrm{In}_{0.13} \mathrm{Ga}_{0.87} \mathrm{As}$. The wafer rotation was stopped during the QD layer growth in order to introduce a gradient in the QD density.

To produce the $\mathrm{p}$-i-n diode structure, a $25 \mathrm{~nm}$-doped layer is grown on top of the membrane, and a $35 \mathrm{~nm}$ n-doped layer is grown in the middle of the membrane. Figure 1(b) shows the band diagram and dc electric field of this structure at zero voltage bias, obtained using a one-dimensional Poisson solver. ${ }^{19}$ The dc electric field across the bottom QD layer remains constant with an applied voltage.

We use three-dimensional finite difference time domain simulations to model the optical cavity mode field for our specific membrane thickness. The $\mathrm{E}_{\mathrm{y}}$ cavity mode electric field amplitude in the $\mathrm{x}-\mathrm{y}$ and $\mathrm{x}-\mathrm{z}$ planes of the L3 photonic crystal defect cavity ${ }^{20}$ is calculated for a $190 \mathrm{~nm}$ thick membrane (Fig. 2). In order to obtain optimal coupling between a single QD and a photonic crystal cavity mode, the QD layer is usually grown in the middle of the membrane. However, in (a)

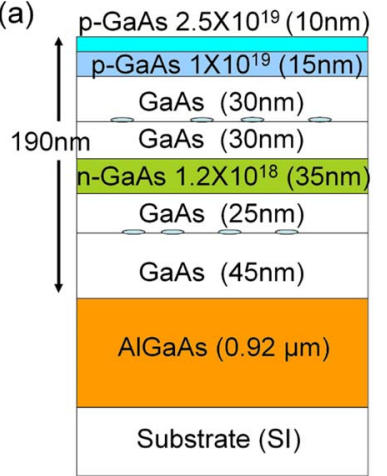

(b)

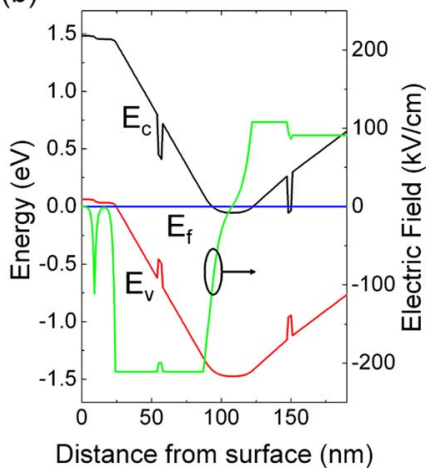

FIG. 1. (Color online) (a) Sample structure for the double QD layer membrane photonic crystals. (b) Simulated band energy diagram of the structure in cross section with air in place of the AlGaAs layer. The conduction band $\left(E_{c}\right)$ and the valence band $\left(E_{v}\right)$ are shown. The Fermi energy $\left(E_{f}\right)$ is zero eV, and the calculated dc electric field is also indicated. 
(a)

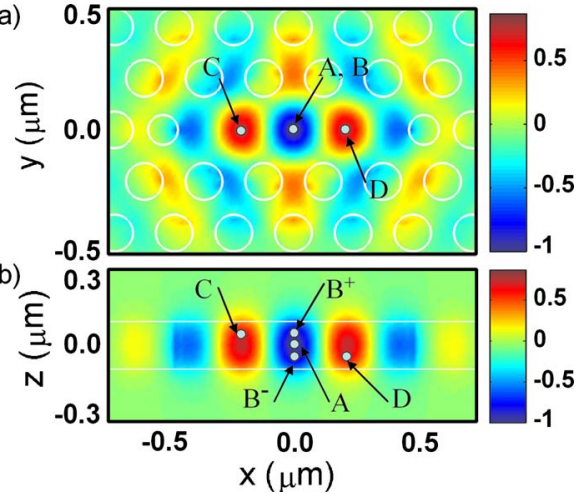

FIG. 2. (Color online) Simulated $E_{y}$ optical field amplitude in the (a) $x-y$ plane and (b) $\mathrm{x}-\mathrm{z}$ plane of the membrane in a L3 photonic crystal defect cavity with a $190 \mathrm{~nm}$ thick membrane. The simulated frequency is 304.12 $\mathrm{THz}(986 \mathrm{~nm})$, and the lattice constant and hole radius are $240 \mathrm{~nm}$ and $74 \mathrm{~nm}$, respectively.

the two-QD-layer scheme, each QD layer needs to be shifted from the center of the membrane. If the QD layer is located at 1/4 of the membrane thickness from the center, the optical field at the location of the QDs is about $85 \%$ at positions $\mathrm{B}^{+}$ and $\mathrm{B}^{-}$and $74 \%$ at positions $\mathrm{C}$ and $\mathrm{D}$ in Fig. 2 of that in the center of the membrane (position A in Fig. 2).

Assuming a QD is located at the maximum optical field intensity location of the L3 photonic crystal cavity, the minimum required quality factor of the cavity is $\sim 3500$ to enable strong coupling. ${ }^{21}$ For the double QD structures considered here, the QD layers are located at about $74 \%-85 \%$ of the maximum cavity mode field in the vertical direction. This corresponds to a required minimum quality factor of about 4000-5000 for reaching strong coupling between a single QD and the cavity mode, assuming the QD is located at the local maximum field location in the lateral direction. If two QDs can be simultaneously coupled to a single cavity mode, strong coupling can be achieved with a factor of $\sqrt{2}$ decrease in the coupling constant ${ }^{22}$ compared to that of a single QDcavity system.

Making good electric contacts to the doped layers is a challenge because of the small layer thicknesses and doping concentrations. To fabricate the electric contacts, the GaAs layer is etched with citric acid/ $\mathrm{H}_{2} \mathrm{O}_{2}$ to expose the n-doped layer. After the etch step, a Ni/AuGe/Ni/Au film is deposited using an electron beam evaporator and annealed to obtain an $\mathrm{n}$-contact metal. The $\mathrm{p}$-contact metal is made by evaporating a Ti/Pt/Au metal on the top of the GaAs membrane.

Alignment marks used as position indicators for the photonic crystals during electron beam lithography are fabricated in parallel with the p-contact metal. The size of each device mesa is $850 \times 400 \mu \mathrm{m}^{2}$. Each device has two $250 \mu \mathrm{m}$ wide metal contacts as shown in Fig. 3(a). After making the mesas, photonic crystals can be fabricated in the region between the $\mathrm{p}$ and $\mathrm{n}$ contacts. The photonic crystal cavity fabrication begins with depositing a $6000 \AA$ thick ZEP520 electron beam resist layer. After electron beam exposure and development, the sample is etched in a $\mathrm{Cl}_{2}$ based inductively coupled plasma reactive ion etching process. L3 photonic crystal cavities were fabricated with several hole sizes and lattice constants in this membrane structure. Quality factors of 3000-5000 were obtained in this sample as shown in Fig. 3(c).

Figure 4 shows the cavity mode $(\lambda \simeq 975.8 \mathrm{~nm})$ and

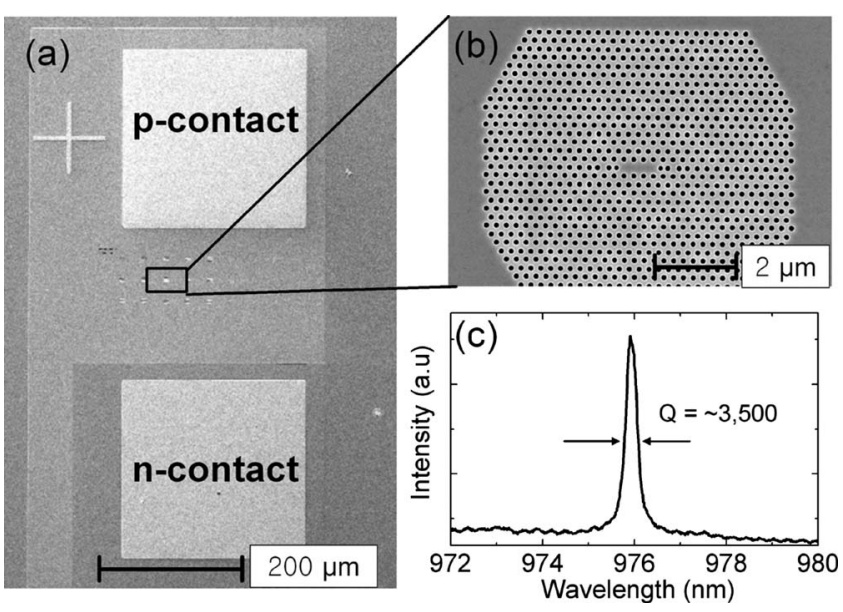

FIG. 3. SEM images of (a) an isolated p-i-n diode device, and (b) a fabricated photonic crystal cavity. (c) Measured cavity mode with Q of $\sim 3500$.

several QD emission lines in the photoluminescence spectrum from one photonic crystal device. The cavity mode is identified by strongly pumping the device such that the QD emission is saturated. Out of many emission lines in the spectrum, about half of the QDs show Stark shift tuning, while the others remain at a constant wavelength. Two QDs (QD1 and QD2) show bright signals due to their proximity to the cavity center and stay at the same frequency during the dc bias voltage scan.

When a dc bias voltage is applied, several QDs show clear Stark shifts over a $1 \mathrm{~nm}$ tuning range. One QD line (QD4) is tuned through resonance with the cavity mode resulting in a slight increase in the overall cavity mode intensity. However, strong coupling is not observed in this device due to the modest quality factor of the cavity and nonoptimal spatial overlap between the QD and the photonic crystal cavity mode.

In order to improve the photonic crystal quality factor, it is necessary to minimize the optical loss due to the free carrier absorption in the dopant layers. This implies finding a compromise between low electrical resistance and low optical loss by choosing an appropriate dopant concentration.

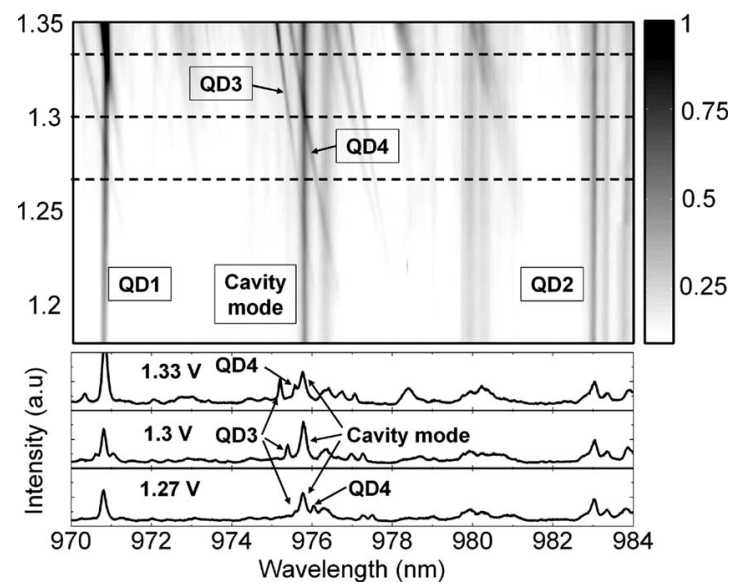

FIG. 4 . Stark shift tuning in a L3 cavity $(Q \simeq 4000)$. The cavity mode is the line at $\lambda=975.8 \mathrm{~nm}$. Two clear QD emission lines are shown at $970.7 \mathrm{~nm}$ (QD1) and $983 \mathrm{~nm}$ (QD2). These QDs are in the bottom QD layer and show no voltage dependence. QDs in the top QD layer (QD3 and QD4) show Stark shift tuning with an applied voltage. The graphs in the bottom show the photoluminescence spectra at various applied voltages $(1.27,1.3$, and $1.33 \mathrm{~V})$. 
By reducing the thickness of the n-doped layer from 35 to $25 \mathrm{~nm}$ and the $\mathrm{n}$-doping concentration from $1.2 \times 10^{18}$ to $1 \times 10^{18} \mathrm{~cm}^{-3}$, we were able to fabricate photonic crystal cavities with quality factors above 10000 with similar I-V characteristic and tuning behavior as the sample presented in this letter.

To achieve strong coupling between a single photonic crystal cavity mode and two separate QDs, the next step would be to apply the optical positioning technique ${ }^{12,13}$ to preselect QDs with promising characteristics. If two QDs with similar emission wavelengths can be found within a certain distance, for instance at positions $\mathrm{C}$ and $\mathrm{D}$ or $\mathrm{B}^{+}$and $\mathrm{B}^{-}$in Fig. 2, ${ }^{10}$ proper photonic crystal cavities can be designed and fabricated around them. Even in the weak coupling regime, the independent tuning method could be useful to generate entanglement between separate QDs. ${ }^{23}$

In summary, by applying an electric bias to only one layer of QDs, we demonstrated that we could tune the emission wavelength of one QD layer independently of the other layer in a photonic crystal cavity. By combining this technique with the optical positioning method, strong coupling of two QDs to a single photonic crystal cavity mode should be feasible.

This work was supported by NSF Grant No. 0901886 and Marie Curie under Grant No. EXT-CT-2006-042580. A portion of this work was done in the UCSB nanofabrication facility, part of the NSF funded NNIN network. S.T. acknowledges financial support from the U.S. Department of Education GAANN grant.

${ }^{1}$ J. P. Reithmaier, G. Sęk, A. Löffler, C. Hofmann, S. Kuhn, S. Reitzenstein, L. V. Keldysh, V. D. Kulakovskii, T. L. Reinecke, and A. Forchel, Nature (London) 432, 197 (2004).

${ }^{2}$ T. Yoshie, A. Scherer, J. Hendrickson, G. Khitrova, H. M. Gibbs, G. Rupper, C. Ell, O. B. Shchekin, and D. G. Deppe, Nature (London) 432, 200 (2004).

${ }^{3}$ E. Peter, P. Senellart, D. Martrou, A. Lemaître, J. Hours, J. M. Gérard, and J. Bloch, Phys. Rev. Lett. 95, 067401 (2005).
${ }^{4}$ K. Hennessy, A. Badolato, M. Winger, D. Gerace, M. Atatüre, S. Gulde, S. Fält, E. L. Hu, and A. Imamoğlu, Nature (London) 445, 896 (2007).

${ }^{5}$ D. Englund, A. Faraon, I. Fushman, N. Stoltz, P. Petroff, and J. Vučković, Nature (London) 450, 857 (2007).

${ }^{6}$ H. Xu, F. W. Strauch, S. K. Dutta, P. R. Johnson, R. C. Ramos, A. J. Berkley, H. Paik, J. R. Anderson, A. J. Dragt, C. J. Lobb, and F. C. Wellstood, Phys. Rev. Lett. 94, 027003 (2005).

${ }^{7}$ D. Bouwmeester, A. Ekert, and A. Zeilinger, The Physics of Quantum Information (Springer, Berlin, 2000).

${ }^{8}$ J. I. Cirac, P. Zoller, H. J. Kimble, and H. Mabuchi, Phys. Rev. Lett. 78, 3221 (1997)

${ }^{9}$ Y. F. Xiao, J. Gao, X. B. Zou, J. F. Mcmillan, X. Yang, Y. L. Chen, Z. F. Han, G. C. Guo, and C. W. Wong, New J. Phys. 10, 123013 (2008).

${ }^{10}$ A. Imamoğlu, S. Fält, J. Dreiser, G. Fernandez, M. Atatüre, K. Hennessy, A. Badolato, and D. Gerace, J. Appl. Phys. 101, 081602 (2007).

${ }^{11}$ A. Badolato, K. Hennessy, M. Atatüre, J. Dreiser, E. Hu, P. M. Petroff, and A. Imamoğlu, Science 308, 1158 (2005).

${ }^{12}$ S. M. Thon, M. T. Rakher, H. Kim, J. Gudat, W. T. M. Irvine, P. M Petroff, and D. Bouwmeester, Appl. Phys. Lett. 94, 111115 (2009).

${ }^{13}$ A. Dousse, L. Lanco, J. Suffczynski, E. Semenova, A. Miard, A. Lemaitre, I. Sagnes, C. Roblin, J. Bloch, and P. Senellart, Phys. Rev. Lett. 101, 267404 (2008).

${ }^{14}$ A. Faraon, D. Englund, I. Fushman, J. Vučković, N. Stoltz, and P. Petroff, Appl. Phys. Lett. 90, 213110 (2007).

${ }^{15}$ A. Laucht, F. Hofbauer, N. Hauke, J. Angele, S. Stobbe, M. Kaniber, G. Bohm, P. Lodahl, M. C. Amann, and J. J. Finley, New J. Phys. 11, 023034 (2009).

${ }^{16}$ S. Mosor, J. Hendrickson, B. C. Richards, J. Sweet, G. Khitrova, H. M. Gibbs, T. Yoshie, A. Scherer, O. B. Shchekin, and D. G. Deppe, Appl. Phys. Lett. 87, 141105 (2005).

${ }^{17}$ S. Strauf, M. T. Rakher, I. Carmeli, K. Hennessy, C. Meier, A. Badolato, M. J. A. Dedood, P. M. Petroff, E. L. Hu, E. G. Gwinn, and D. Bouwmeester, Appl. Phys. Lett. 88, 043116 (2006).

${ }^{18} \mathrm{~K}$. Hennessy, A. Badolato, A. Tamboli, P. M. Petroff, E. Hu, M. Atatüre, J. Dreiser, and A. Imamoğlu, Appl. Phys. Lett. 87, 021108 (2005).

${ }^{19}$ G. L. Snider, Computer Program: 1D Poisson/Schrodinger: A Band Diagram Calculator. (http://www.nd.edu/ gsnider, University of Notre Dame, Notre Dame, Indiana).

${ }^{20}$ Y. Akahane, T. Asano, B.-S. Song, and S. Noda, Nature (London) 425, 944 (2003).

${ }^{21}$ G. Khitrova, H. M. Gibbs, M. Kira, S. W. Koch, and A. Scherer, Nat Phys. 2, 81 (2006)

${ }^{22}$ P. F. Herskind, A. Dantan, J. P. Marler, M. Albert, and M. Drewsen, Nat Phys. 5, 494 (2009).

${ }^{23}$ E. Waks and J. Vuckovic, Phys. Rev. Lett. 96, 153601 (2006). 